\title{
ANALYSIS AND EXPERIMENTS OF EMBEDDED GRIPPING MECHANISM USED IN LARGE-SCALE TOOLS HOLDING UP PILE FOUNDATION IN OCEAN
}

\author{
Zhuo Wang \\ Bo Zhang \\ Tao Wang \\ Harbin Engineering University, China
}

\begin{abstract}
With the rapid development of the marine economy and continuous improvement of the industry, the scale of the offshore engineering is increasing. This raises interest in studying, theoretically and experimentally, gripping and bearing mechanisms for large-scale holding and lifting tools used in foundation pile installations. In this paper, the embedded gripping mechanism is studied based on the theory of elastic-plastic mechanics. The embedded and bearing performance of the tooth is simulated and the influence factors are studied. In addition, the device used in the simplified embedded experiment on the tooth of the embedded block is designed. The relationship between embedded depth, load, and tooth profile angle is identified and validated. Meanwhile, the embedded performance of linear and ring type teeth is compared experimentally in order to select the suitable type of tooth for various situations. This comparison makes the basis for designing an upending gripper for the marine pile foundation, which can realize the operation of holding the pile to prevent its falling.
\end{abstract}

Keywords: pile foundation; gripping; embedded mechanism; embedded depth; upending gripper

\section{INTRODUCTION}

With the development of economy, the demand for energy increases. The exploitation of offshore oil and wind becomes an important direction of energy development $[1,2]$. Underwater installation of marine foundation piles is one of the key technologies used in installation of offshore platforms and wind turbines [3]. Since the marine foundation piles have several characteristic features, such as large diameter, length, and weight, large-scale holding and lifting tools are used for their installation [4]. In this context, it is advisable to study, theoretically and experimentally, gripping and bearing mechanisms for large-scale holding and lifting tools used for installation of foundation piles in offshore areas.

There are numerous foreign large-scale holding and lifting tools, but their specific principles and research methods are seldom available $[5,6,7]$. In China, many domestic scholars have done theoretical and experimental research in this area
$[8,9,10]$. The contact pressure and extrusion conditions of the pressing block tooth have been obtained based on the finite element analysis. In 2006, an optimal design was introduced into the structure of the pressing block tooth for underwater skirt pile gripper by some scholars from Harbin Engineering University. In 2007, Liang, F.H. et al. of Tianjin University proposed making a tooth or groove on the piston surface to increase friction. In that paper, the finite element analysis was used to choose the number of hydraulic pressure vats distributed in the pile gripper [11]. In 2008, Jiang, Y.C. et al. of Tianjin University put forward a design of the controlled friction skirt pile gripper, and used ANSYS software to obtain the relationship between stress, strain, and force for different pile diameters and wall thickness [12]. In 2009, Li, H.L. et al. of CNOOC completed the experimental examination of mechanical performance of the clamping block [13]. The ultimate load experiment was concluded with the relation between the clamping force and the maximum lifting force 
for clamping blocks made of different materials. In 2011, the mechanical model of contact between pressing block teeth and a steel pile was developed by Jiang, P.R. and Fang, X.M. of Harbin Engineering University [14]. The mechanical performance of the pressing block teeth and the steel pile was examined. In 2013, Li, T.Q. of CNOOC worked out the structural design of the suspension tool. He also analyzed variations of the clamping force, which increased with the increase of the angle between the pile and the horizontal plane [15]. The clamping performance of the Pile Pending Device (PPD) was researched by the company IHC in 2015 [16]. The conclusion from their research was that when the number of uniformly distributed wedge assemblies covers more than $50 \%$ of the circumference of the base ring, the clamping effect is better [17].

Up to the present, most researches have focused on the principle and feasibility of large-scale holding and lifting tools, and on contact problems between the pressing block teeth and the steel pile. Another research issue was optimization of pressing block tooth type via the finite element analysis and experimental validation. A few researches investigated damages to the foundation piles and their bearing capacity introduced by the embedded gripping mechanism. In this paper, the damage to the foundation piles is researched in the simplified experiment on the tooth of the embedded block. Moreover, the embedded performance of linear and ring type teeth is compared. The embedding process performed with the embedded gripping mechanism is analyzed using the finite element simulation.

\section{SIMPLIFIED METHOD OF EXPERIMENT ON EMBEDDED BLOCK TOOTH}

As Fig. 1 shows, the device used in the simplified experiment on the tooth of the embedded block was designed not only to simulate the force acting between the tooth and the pile, but also to examine the pile deformation and damage.

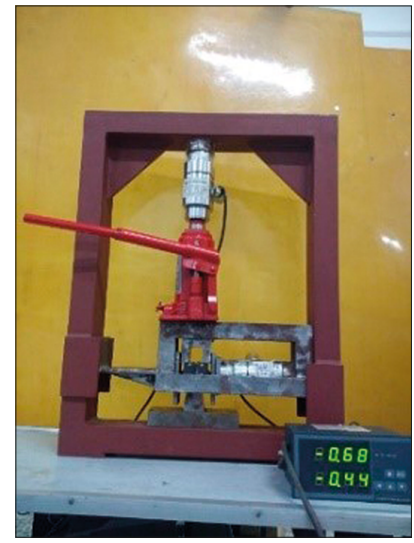

Fig. 1. Device used in simplified embedded experiment

In practice, the diameter of the pile is very large, compared to the size of the embedded block. Therefore, plates were used in the experiment instead of piles, as shown in Fig. 2. When measuring the embedded depth, the material of the used plates was Q235, the yield strength of which is relatively low and the embedded depth was larger than that of the pile material. As shown in the figure, different embedded blocks with different types of teeth and tooth profile angles were examined to find the relationship between the embedded performance and the tooth type and profile angle.

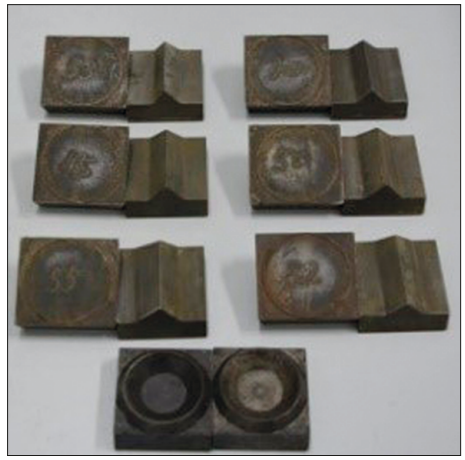

Fig. 2. Plates of piles and embedded block

During the experiment, the embedded block was placed on the wedge-shaped slider which was installed respectively on both sides of the fixed wedge block, as shown in Fig. 3.

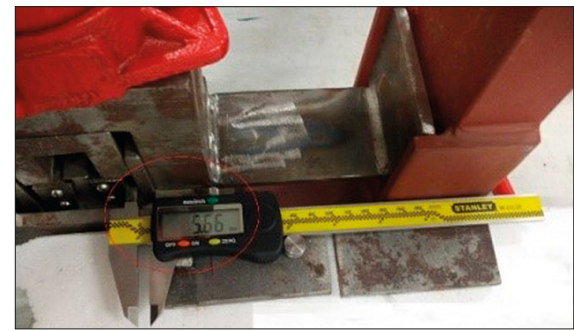

Fig. 3. Diagram of indirect measurement of embedded depth

The plate representing the pile was placed in the main framework to contact with the tooth of the embedded block. The hydraulic jack was installed on the main framework to apply load, which then was measured by the pressure sensor. The embedded depth was calculated by measuring the distance between the wedge-shaped slider and the pile plate before and after the experiment. To extend the range of generality of the results of the experiment, different embedded blocks with different tooth types and profile angles were used.

\section{RESULTS OF THE EXPERIMENT ON EMBEDDED BLOCK TOOTH}

As shown in Tab. 1, in the simplified experiment on the tooth of the embedded block, the embedded blocks of the same linear tooth type but with different tooth profile angles were examined under different pressures. The embedded depth corresponding to each load step was recorded. The average value was obtained from the data of several experiments. Finally, the curve representing the relationship between the load and the embedded depth was drawn.

When the tooth profile angle was the same, the load increase resulted in the increase of the embedded block depth. When the load was the same, the increase of the tooth profile angle resulted in the decrease of the embedded block depth, as shown in Fig. 4. 
Tab. 1. .Embedded depth of different angle of tooth profile

\begin{tabular}{|c|c|c|c|c|c|}
\hline $\begin{array}{c}\text { Angle } \\
\text { of tooth } \\
\text { profile } \\
\left({ }^{\circ}\right)\end{array}$ & $\begin{array}{c}\text { Load } \\
(\mathrm{T})\end{array}$ & $\begin{array}{l}\text { Emb- } \\
\text { edded } \\
\text { depth } \\
(\mathrm{mm})\end{array}$ & $\begin{array}{c}\text { Angle } \\
\text { of tooth } \\
\text { profile } \\
\left({ }^{\circ}\right)\end{array}$ & $\begin{array}{c}\text { Load } \\
(\mathrm{T})\end{array}$ & $\begin{array}{l}\text { Emb- } \\
\text { edded } \\
\text { depth } \\
(\mathrm{mm})\end{array}$ \\
\hline 75 & $\begin{array}{l}1.20 \\
1.50 \\
1.60 \\
1.68 \\
1.78 \\
2.02 \\
2.09 \\
2.44 \\
2.60 \\
2.83 \\
3.00 \\
3.17 \\
3.47\end{array}$ & $\begin{array}{l}0.12 \\
0.20 \\
0.23 \\
0.25 \\
0.28 \\
0.31 \\
0.33 \\
0.35 \\
0.38 \\
0.40 \\
0.42 \\
0.45 \\
0.47\end{array}$ & 85 & $\begin{array}{l}1.20 \\
1.48 \\
1.62 \\
1.72 \\
1.84 \\
2.10 \\
2.20 \\
2.44 \\
2.67 \\
2.80 \\
2.96 \\
3.36 \\
3.55\end{array}$ & $\begin{array}{l}0.10 \\
0.16 \\
0.18 \\
0.20 \\
0.22 \\
0.26 \\
0.28 \\
0.31 \\
0.33 \\
0.35 \\
0.38 \\
0.42 \\
0.44\end{array}$ \\
\hline 95 & $\begin{array}{l}1.15 \\
1.35 \\
1.50 \\
1.69 \\
1.80 \\
2.02 \\
2.36 \\
2.54 \\
2.69 \\
2.97 \\
3.14 \\
3.30 \\
3.55\end{array}$ & $\begin{array}{l}0.08 \\
0.11 \\
0.14 \\
0.16 \\
0.18 \\
0.21 \\
0.24 \\
0.25 \\
0.27 \\
0.30 \\
0.32 \\
0.34 \\
0.37\end{array}$ & 105 & $\begin{array}{l}1.18 \\
1.48 \\
1.57 \\
1.67 \\
1.81 \\
2.07 \\
2.38 \\
2.52 \\
2.65 \\
2.95 \\
3.13 \\
3.40 \\
3.53\end{array}$ & $\begin{array}{l}0.06 \\
0.09 \\
0.11 \\
0.12 \\
0.13 \\
0.16 \\
0.18 \\
0.20 \\
0.22 \\
0.24 \\
0.27 \\
0.29 \\
0.30\end{array}$ \\
\hline
\end{tabular}

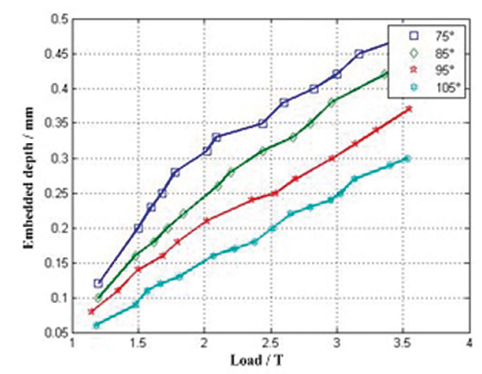

Fig. 4. Curve between the load and the embedded depth

During the experiment, no embedded block breakup was recorded, as shown in Fig. 5. The indentation of the embedded block for the tooth profile angle of $75^{\circ}$ was deep and narrow, while for the angle of $105^{\circ}$ it was shallow and wide. In practice, deep and narrow indentation has higher stress concentration, therefore a larger tooth profile angle should be chosen, provided that the bearing load condition is met.

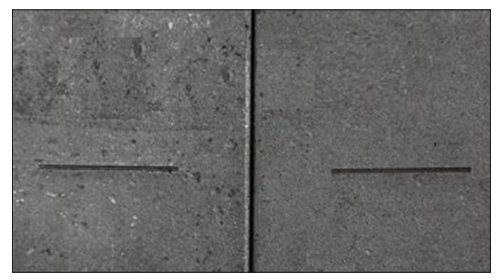

Fig. 5. Indentation of embedded blocks

The embedded performance of the linear and ring type teeth was compared experimentally. The embedded blocks with the same tooth profile angles but of different tooth type were examined under the same pressure. The embedded depth corresponding to each load step was recorded.
Tab. 2. Embedded depth of different type of the tooth

\begin{tabular}{|c|c|c|c|c|c|}
\hline $\begin{array}{l}\text { Linear } \\
\text { type of } \\
\text { tooth }\end{array}$ & $\begin{array}{c}\text { Load } \\
(\mathrm{T})\end{array}$ & $\begin{array}{l}\text { Emb- } \\
\text { edded } \\
\text { depth } \\
(\mathrm{mm})\end{array}$ & $\begin{array}{l}\text { Ring } \\
\text { type of } \\
\text { tooth }\end{array}$ & $\begin{array}{c}\text { Load } \\
(\mathrm{T})\end{array}$ & $\begin{array}{l}\text { Emb- } \\
\text { edded } \\
\text { depth } \\
(\mathrm{mm})\end{array}$ \\
\hline & 1.20 & 0.10 & & 1.37 & 0.09 \\
\hline & 1.48 & 0.16 & & 1.50 & 0.11 \\
\hline & 1.62 & 0.18 & & 1.61 & 0.13 \\
\hline & 1.72 & 0.20 & & 1.74 & 0.14 \\
\hline & 1.84 & 0.22 & & 1.93 & 0.15 \\
\hline & 2.10 & 0.26 & & 2.05 & 0.17 \\
\hline & 2.20 & 0.28 & & 2.26 & 0.19 \\
\hline & 2.44 & 0.31 & & 2.48 & 0.21 \\
\hline & 2.67 & 0.33 & & 2.66 & 0.23 \\
\hline & 2.80 & 0.35 & & 2.77 & 0.24 \\
\hline & 2.96 & 0.38 & & 2.98 & 0.27 \\
\hline & 3.36 & 0.42 & & 3.36 & 0.31 \\
\hline & 3.55 & 0.44 & & 3.50 & 0.32 \\
\hline
\end{tabular}

The experimental data shown in Tab. 2 reveal that the embedded depth of the ring type tooth is significantly smaller than that of the linear type tooth. The reason is that the contact length of the ring type tooth is longer than that of the linear type tooth, consequently the damage of the plate is smaller under the same load.

When the parallelism between the tooth and the pile plate was deteriorated in the experiment, the phenomenon of uneven contact occurred. Since the contact trace of the linear type tooth was a straight line, this reduced the phenomenon of uneven contact to a certain extent, as shown in Fig. 6.

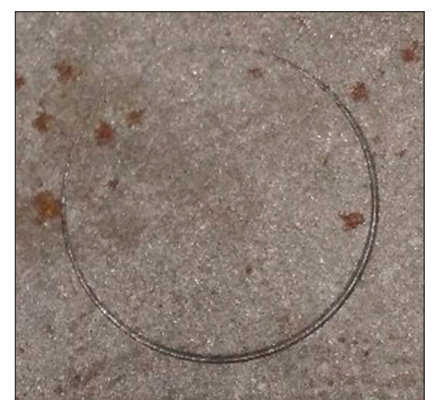

Fig. 6. Uneven indentation of embedded blocks

In practice, the plate damage caused by the ring type tooth was small, and the ring type tooth could withstand forces in all directions. In cases when the load changes in various directions, the ring type tooth should be chosen. The ring type tooth is structurally simple and convenient in processing. When the parallelism between the tooth and the pile plate is poor but the direction of load does not change, the linear type tooth should be chosen. 


\section{ALYSIS AND DISCUSSION ON THE EMBEDDED GRIPPING MECHANISM}

The analysis on the embedded mechanism between the embedded block tooth and the pile. The embedded block increased the friction and provided the bearing capacity for the pile by embedding into the pile. The embedded mechanism between the tooth of the embedded block and the pile had the following characteristics:

1) Compared to the size of the pile, the size of the tooth was very small and sharp.

2) The stress in the pile caused by the tooth was larger than the yield strength of the pile, as a consequence of which the tooth embedded into the pile.

When the pile was clamped, it formed the interactive compression between the tooth and the pile. Therefore, the pile yielded and the tooth embedded into the pile. With the increase of the embedded depth, the metal of the pile slipped on the face of the tooth and the contact area increased. Once the limit load was reached, the plastic deformation of the pile stopped and the metal stopped slipping. Meanwhile, the tooth stopped embedding into the pile and the process reached the equilibrium state.

The embedding process between the tooth and the pile is a complex contact process. It can be simplified to the plane contact problem. The formula of the embedded depth can be obtained using the slip line method.

As shown in Fig. 7 (a) and (b), the embedded block with tooth profile angle $2 \gamma$ was under the pressure $N_{n}$ to embed into the pile. The embedded depth was $t$, and the normal stress of the contact surface was $q$. Considering the frictional force of the contact surface, the friction shear stress was $k$. a)

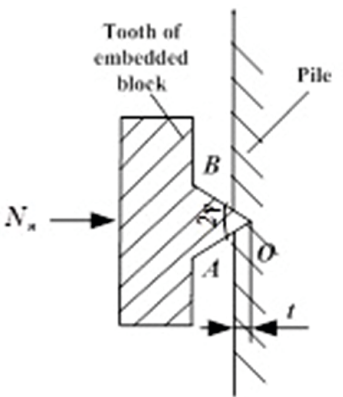

b)

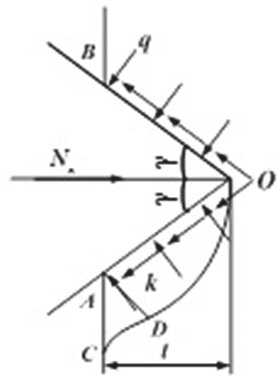

Fig. 7. Force diagram between the tooth and the pile

Due to the symmetry of the problem, the direction of the pile slip line was determined by examining half of the tooth. $A D$ was the slip line of the area $A D C$, according to the direction of the maximum principal stress. Then, $A O$ was the slip line of the area $A O D$, according to the direction of the maximum shear stress.

The average stress on the boundaries $A C$ and $A O$ can be illustrated by the boundary normal stress component $\sigma_{n}$, the yield stress of pile material $\tau_{s}$, the angle $\theta$ between the slip line and the $x$-axis, and the angle $\varphi$ between the boundary normal and the $x$-axis.

$$
\begin{gathered}
\sigma=\sigma_{n}+\tau_{s} \sin 2(\theta-\varphi)=-\tau_{s} \\
\sigma=\sigma_{n}+\tau_{s} \sin 2(\theta-\varphi)=-q
\end{gathered}
$$

Along with the slip line, the equation can take the form of the Hencky equation:

$$
\sigma+2 k \theta=C_{\beta}
$$

By considering (1), (2) and (3), the equations can be set up as:

$$
\left\{\begin{array}{l}
-\tau_{s}+2 \tau_{s}\left(-\frac{3 \pi}{4}\right)=C_{\beta 1} \\
-q+2 \tau_{s}\left(-\frac{\pi}{2}+\gamma\right)=C_{\beta 2}
\end{array}\right.
$$

If the slip line is straight, $\sigma, \theta, C_{\alpha}$, and $C_{\beta}$ are constant along the line. Consequently, $C_{\beta 1}=C_{\beta 2}=$ constant.

Therefore, the equation can take the form:

$$
q=\tau_{s}\left(\frac{\pi}{2}+2 \gamma+1\right)
$$

Based on the known condition, the force equilibrium equation was set up:

$$
N_{n}=\left(l_{O A}+l_{O B}\right)\left(q \sin \gamma+\tau_{s} \cos \gamma\right)
$$

where, $l_{O A}=l_{O B}=\frac{t}{\cos \gamma}$ 。

The formula of the embedded depth can be obtained after substituting (5) into (6):

$$
t=\frac{N_{n}}{2 \tau_{s}\left(\frac{\pi}{2} \tan \gamma+2 \gamma \tan \gamma+\tan \gamma+1\right)}
$$

The curved surface shown in Fig. 8 represents the relationship between the embedded depth of the block, the applied load, and the used tooth profile angle.

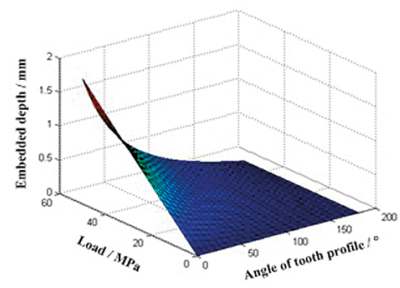

Fig. 8. Curve of the embedded depth

It reveals that the embedded block depth increases with the increase of load and the decrease of tooth profile angle. This result is consistent with the experiment, on the whole. In order to avoid pile failure and the decrease of ultimate bearing capacity of the tooth caused by improper embedded depth, the embedded blocks with appropriate tooth profile angles should be chosen, and appropriate loads should be applied.

The simulation analysis on the embedding process of embedded block tooth. The embedding process of the tooth 


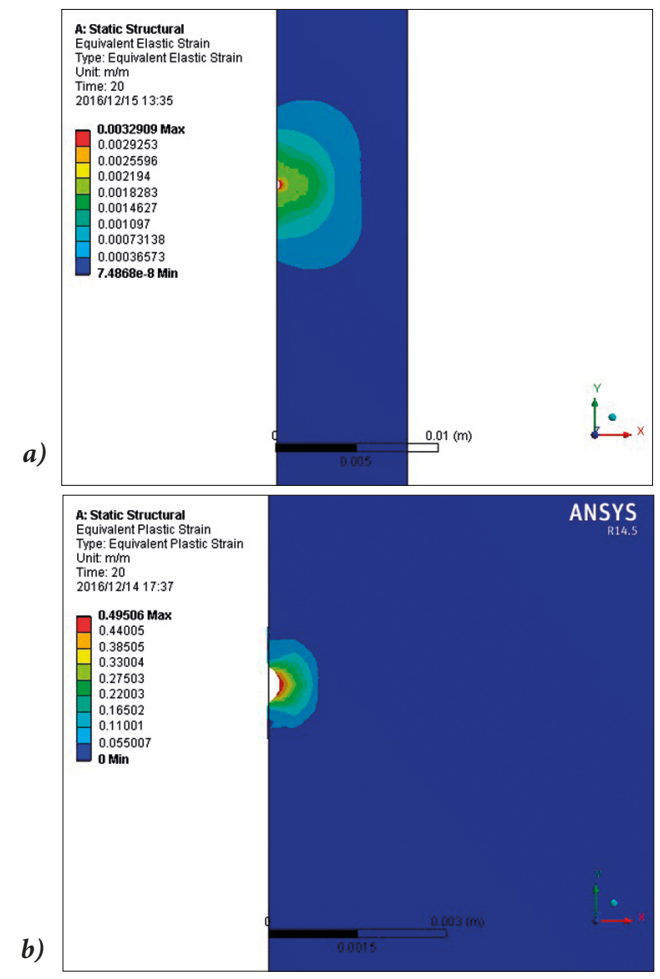

Fig. 9. Elastic and plastic strain of the pile

belongs to the category of nonlinear processes, which not only require advanced and time-consuming calculations, but are also difficult to converge. Therefore, the finite element models of the tooth and the pile were described by plane elements. To improve the calculation accuracy and speed, the mesh was refined at the location where the tooth embedded into the pile, while in other places, a coarsened grid was used. The contact model between the tooth and the pile was established, in which the tooth and the pile were the contact body and the target body for each other.

The simulation analysis was carried out with the tooth of the embedded block and the pile. In the simulation, the embedded block with tooth profile angle of $90^{\circ}$ was under the pressure of $30 \mathrm{MPa}$. To find the embedded depth of the tooth, the pile was analyzed separately.

The pile generated elastic deformation at the location where the tooth embedded into it under the applied load. When the elastic deformation reached the maximum, the pile entered the stage of plastic deformation. The elastic and plastic strain regions are shown in Fig. 9. The region of elastic strain is obviously larger than that of plastic strain. The maximum of elastic strain was 0.0033 , and the maximum of plastic strain was 0.495 , as shown in the figure.

The equivalent stress of the pile gradually increased along the direction of thickness at the location where the tooth was embedded under the applied load, as shown in Fig. 10. The equivalent stress reached a maximum of $657.18 \mathrm{MPa}$ and was above the yield strength of the pile material, as a consequence of which the tooth embedded into the pile to the depth of $0.186 \mathrm{~mm}$.

A small axial displacement was subjected to the top of the pile. The ultimate bearing capacity of the tooth was obtained

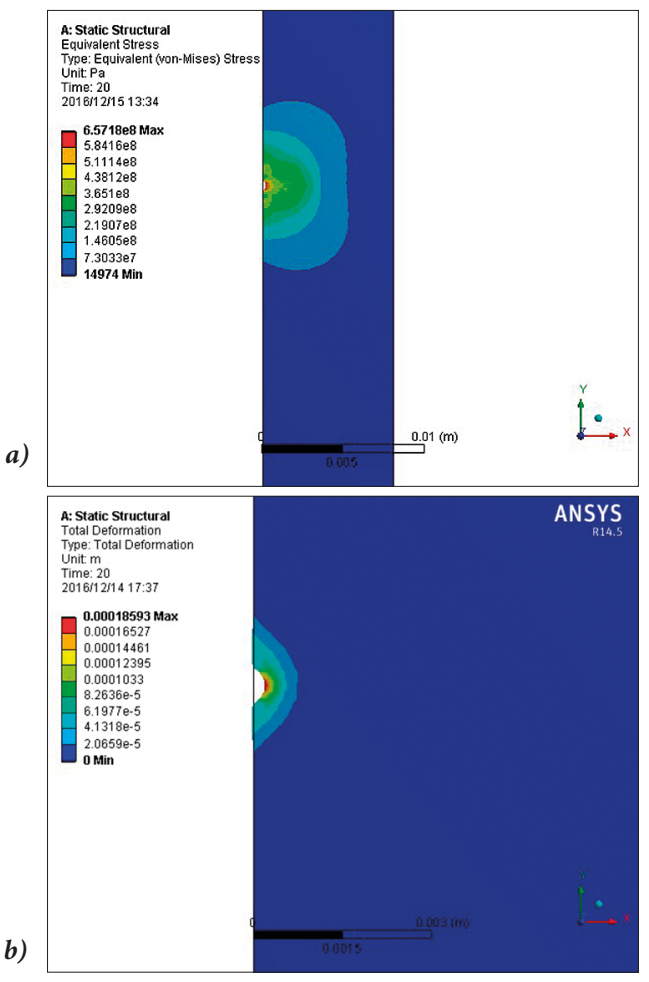

Fig. 10. Equivalent stress of the pile and embedded depth

by applying the constraint force in the simulation. It was equal to $313.93 \mathrm{kN}$ under the pressure of $30 \mathrm{MPa}$.

Similarly, different loads were applied to the embedded blocks with different tooth profile angles to simulate the embedding process. The curved surface of the embedded depth, load, and tooth profile angle obtained from the simulations is shown in Fig. 11.

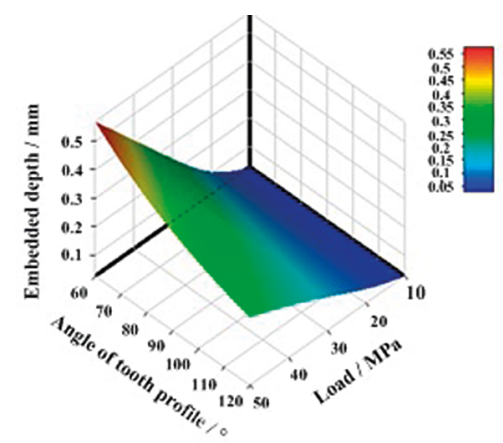

Fig. 11. Curve of the embedded depth

Changes of the theoretical value, the simulation value, and the experimental value of the embedded depth were basically the same. However, the experimental value was larger than the theoretical value and the simulation value. The theoretical value, which was studied by the slip line method, was closer to the experimental value. The embedded depth measured in the experiment included not only the deformation of the pile, but also the elastic deformation of the tooth. Besides, the embedded depth was obtained by indirect measurement in the experiment. The measurement errors made the experimental value larger than the theoretical value and the simulation value. 
Design of the pile upending gripper. During pile installation, the pile upending gripper clamps the pile and turns it from horizontal to vertical position. This reduces difficulties and improves the efficiency of the installation process. When the gripper upends the pile, it clamps the pile to prevent pile dropping.

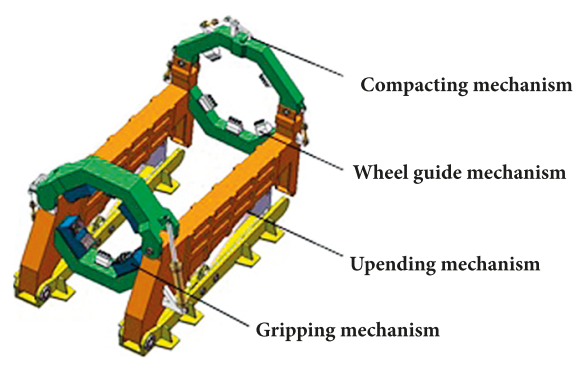

Fig. 12. Schematic of the pile upending gripper

As shown in Fig. 12, the pile upending gripper was designed by using the embedded gripping mechanism to increase friction and clamping force. The pile upending gripper includes gripping mechanism, wheel guide mechanism, compacting mechanism, and upending mechanism.

When the pile upending gripper upends the pile, the gripping mechanism clamps the pile in order to prevent pile slipping. The gripping mechanism is mainly composed of clamping jaw, frame connecting jaw, jaw opening hydraulic cylinder, clamping hydraulic cylinders, embedded blocks, boxes, and wheels. The clamping hydraulic cylinder piston rods and the mounting plate of the embedded blocks are in threaded connection. The embedded blocks are uniformly arranged in the groove of the mounting plate, as shown in Fig. 13.

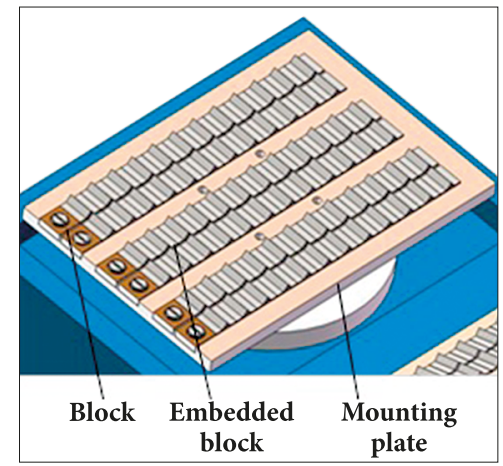

Fig. 13. Arrangement of embedded blocks

When the clamping hydraulic cylinders clamp the pile, the blocks embed into the pile. The resistance to bend and shear is produced to increase friction.

\section{CONCLUSIONS}

The paper analyses the embedded gripping mechanism and the damage done by it to the foundation piles. Based on that, the pile upending gripper is proposed. The conclusions are as follows: the damage to the foundation piles is very small when using the embedded gripping mechanism which can effectively increase friction. With the increase of load and the decrease of tooth profile angle, the embedded block depth increases. The embedding performance of the linear and ring type teeth is different. Therefore, they should be used in different situations.

\section{ACKNOWLEDGEMENT}

The research was financed by the National Natural Science Foundation of China, within the framework of the project entitled "Research on ultimate bearing capacity and parametric design for the grouted clamps strengthening the partially damaged structure of jacket pipes" (NSFC Grant No. 51879063) and "Research on analysis and experiments of gripping and bearing mechanism for large-scale holding and lifting tools on ocean foundation piles" (NSFC Grant No: 51479043).

\section{REFERENCES}

1. Sarkar, A., Gudmestad, O. T. Installation of monopiles for offshore wind turbines - by using end-caps and a subsea holding structure. ASME 2011 International Conference on Ocean, Offshore and Arctic Engineering, 2011: 309-315.

2. Sarkar, A., Gudmestad, O. T. Study on a new method for installing a monopile and a fully integrated offshore wind turbine structure. Marine Structures, 2013, 33(10): 160-187.

3. Hezarjaribi, M., Bahaari, M. R., Bagheri, V., et al. Sensitivity analysis of jacket-type offshore platforms under extreme waves. Journal of Constructional Steel Research, 2013, 83: 147-155.

4. Wang, W., Yang, M., Review and discussion on key technologies in foundation design of offshore wind power, Journal of Hydroelectric Engineering, 2012,31(06): 242-248.

5. Natskår, A., Moan, T., Alvær, P. Uncertainty in forecasted environmental conditions for reliability analyses of marine operations. Ocean Engineering, 2015, 108(2): 636-647.

6. http://www.knudehansen.com/references/offshoreoffshore-wind-vessels/mono-pile-upending-trolley/.2017.

7. http://www.ihchs.com/wind/deck-equipment/pile-guidingand-positioning-frame/.2017.

8. Sun, Z. J., Meng, Q.X., Zhou, D. Contact finite element analysis of the pressing block tooth in underwater skirt pile gripper of offshore oil platform. Machinery, 2006, (12): 1-4.

9. Sun, Z. J., Meng, Q.X., Zhou, D. Structure optimization of the pressing block tooth in underwater skirt pile gripper of offshore oil platform. Machine Design and Research, 2006, (06): 102-105. 
10. Sun, Z.J., Meng, Q.X., Zhou, D. Contact nonlinear finite element analysis on the pressing block tooth in underwater skirt pile gripper. Journal of Petrochemical Universities, 2006, (03): 72-75.

11. Liang, F.H., Yan, X.G., Zheng, W. Mechanical analysis of jacket pile gripper and choice of the number of hydraulic pressure vats. The Ocean Engineering, 2007, (01): 82-87.

12. Jiang, Y.C., Tian, Y.F., Yang, S.G., The skirt pile gripper design of jacket platform. China Offshore Platform, 2008, (05): $19-22+26$.

13. Li, H.L., Yu, W.T., Wang, L.Q., et al. Experimental research on clamping block of underwater leveling equipment. China Offshore Oil and Gas, 2009, (01): 57-60.

14. Jiang, P.R., Fang, X.M. Analysis and experimental study grip function of an underwater skirt the gripper. Journal of Harbin Engineering University, 2011, (09): 1228-1232.

15. Li, T.Q. Research of unilateral clamping lifting tool of great steel tube stake at sea. Modern Manufacturing Engineering, 2013, (08): 79-82+59.

16. Mulderij, K. J. Pile upending device: WO, WO/2014/ 084738[P], 2014.

17. Stefan, A., Constantin, D., Grigore, L. S. Aspects of kinematics and dynamics of a gripping mechanism. Electronics, Computers and Artificial Intelligence (ECAI), 7th International Conference on, 2015: WF-1-WF-4.

\section{CONTACT WITH THE AUTHORS}

\author{
Zhuo Wang \\ e-mail:2958219945@qq.com
}

Harbin Engineering University Road Nantong 145, District Nangang 150001 Harbin

China 